\title{
INCOMPLETE PREFERENCES AND THE PREFERENCE FOR FLEXIBILITY*
}

\author{
Ricardo Arlegi \\ Universidad Pública de Navarra \\ Departamento de Economía \\ Campus Arrosadia s/n -31006 Pamplona (Spain) \\ e-mail: rarlegi@ si.upna.es. fax: 34-948-169721.phone: 34-948-169344. \\ December 1998
}

\begin{abstract}
Sometimes individual agents display in their decisions a preference for having more opportunities to choose from. In this paper this fact is interpreted from a preference for flexibility approach, which links with the hypothesis that there is some uncertainty or vagueness in the decision maker's preferences. We define an asymmetric (but not necessarily transitive, or complete) preference on a finite universe of alternatives, $X$, to express that vagueness. Taking this preference as a reference, the notion of preference for flexibility is described by means of the axiomatic characterization of a class of binary relations over the possible subsets of $X$, which are interpreted as opportunity sets. Subsequently, we demonstrate the relationship between the results and the representation theorem of preference for flexibility proposed by Kreps [10]. Finally, it is shown how some special orderings of the related literature could be interpreted as elements of the class characterized here.

* This work was possible thanks to the financial support of the Comisión Interministerial de Ciencia y Tecnología (SEC96-0858). I appreciate comments and suggestions by Jorge Nieto, which were necessary for the completion of this paper. This paper has also benefited from the comments of Carmen Herrero, Mari Carmen Marco, and Jose Mari Aizpurua. The usual caveat applies.
\end{abstract}




\section{Introduction}

In certain problems of individual choice, the decision maker has only provisional, or not completely made up preferences among the alternatives at her disposal. When this happens, and if it is possible to postpone the final choice, it is plausible to think that she would like the chance to maintain bigger opportunity sets to choose from. This problem, where future preferences are uncertain, has been called preference for flexibility in the related literature (see Koopmans [9], Arrow [2], and specially Kreps [10]). According to the last author, in such situations, preferences on opportunity sets satisfy that " $a$ set is at least as good as all of its subsets, but may not satisfy 'revealed preference', the union of two sets may be strictly preferred to each one taken separately" (Kreps [10,p.565]). This motivates him to provide a rationalization of this type of behavior by means of contingent utility functions.

In relation to the preference for flexibility literature, but with a different motivation, there has in recent years been increasing debate over the individual value of freedom of choice (see Pattanaik and Xu [13], Bossert [4], Bossert, Pattanaik and Xu [5], Gravel [6], Klemisch-Ahlert [8], Puppe [14,15], Sen $[17,18,19]$ or Suppes [20] among others). In most cases these works turn, more or less exclusively, to the intrinsic value of freedom of choice, according to which an enlargement of the set of opportunities always implies a strict improvement of individual welfare (see Sen [18]); that is, the condition satisfied is that, for any opportunity set $A$, and for any alternative $x$ not belonging to $A$, the set $A \cup\{x\}$ is strictly better than $A$. The desire for a greater range of choice is in this case independent of the uncertainty of the preferences. In general, the notion of freedom of choice is formalized by means of the axiomatic characterization of preferences on opportunity sets which reflects such a desire for freedom of choice (it is better to have more to choose from), while simultaneously being consistent with the ex-ante preferences on the individual alternatives, or basic preferences (it is better to have good alternatives to choose from than bad ones).

In contrast with the above mentioned works, the current work returns to the idea of preference for flexibility. According to the interpretation of the term "preference for flexibility", an enlargement of the 
opportunity set should strictly improve the agent's welfare only in some cases; in particular, only if it allows her finally to select a better alternative than any other she could have selected before the enlargement. In this case, it is not generally true that any opportunity added to a given set necessarily implies a strict improvement. This hypothesis leads us naturally to examine the agent's preferences on the alternatives individually considered, that is, it seems reasonable to analyze those attributes of the basic preferences, which give rise to the discrimination between alternatives whose availability is valued and those whose availability is not.

Therefore, the formalization of the idea of preference for flexibility presented here is in some way related both with the freedom of choice literature and with works on preference for flexibility: We take the axiomatic methodology of the freedom of choice literature, but with a "preference for flexibility" motivation. That is, we axiomatically characterize orderings over opportunity sets coherent with the basic preference but, in our case, since that preference may be uncertain, it has a particular structure (in particular, it is supposed to be only asymmetric, and may be clearly incomplete). On the other hand, our description of the concept of preference for flexibility differs to that of Kreps, whose model lacks any formal allusion to an underlying binary relation on $X$.

This approach enables us to show that some paradigmatic rankings of the freedom of choice literature, such as the cardinalist one (see Pattanaik and $\mathrm{Xu}$ [13]), or the leximax (see Bossert, Pattanaik and Xu [5]), can be axiomatically obtained as coherent with the preference for flexibility idea; that is, sometimes the apparent intrinsic value of freedom of choice, provided by the mere availability of more alternatives, may mask some uncertainty as to future preferences. On the other hand, we show the formal link between both types of formalization of the preference for flexibility concept (the axiomatic characterization proposed here, and Kreps' representation theorem).

In order to illustrate the differences between the preference for flexibility idea and the freedom of choice motivation, let us consider the following example: Let us imagine a student who can choose between studying Law or Economics at her local university. If, afterwards, this university offers her the additional option to study Medicine, it seems that her welfare increases in as much as she has more 
opportunities at her disposal, and in consequence, enjoys more flexibility in choosing a career. However, let us suppose that she loves Economics and would never prefer to be a doctor to being an economist. In this case, would her capacity to decide be affected by the incorporation of the third option? We could reasonably assert that the opportunity set $\{(\mathrm{E})$ conomics, $(\mathrm{L}) \mathrm{aw},(\mathrm{M})$ edicine $\}$ provides her with the same flexibility to choose as $\{\mathrm{E}, \mathrm{L}\}$, because she will never take the alternative $(\mathrm{M})$ if $(\mathrm{E})$ is available. In contrast, let us imagine another student who prefers (E) or $(\mathrm{L})$ to $(\mathrm{M})$, but who is not yet absolutely certain of his tastes. In this case, although he values (E) and (L) more highly than (M), it might be reasonable to think that he would like to keep the third option open if possible; so that, in such circumstances, the enlargement of the set could plausibly be considered as an improvement in terms of flexibility.

Examples such as the one above suggest that, in fact, the analysis of the agent's basic preferences, and especially in so far as the certainty of these is concerned, is a necessary, or at least, a natural starting point for the correct study of her desire for flexibility. A first simple step in assessing the importance of these factors is to distinguish, among the binary relations of preference on the alternatives, between those which are absolutely certain in nature (the decision maker has no doubt that she prefers $x$ to $y$, and is sure that she will never choose $y$ if she can choose $x$ ), and those which are not so certain (perhaps the agent prefers $x$ to $y$, but is not absolutely sure about her tastes; she cannot claim that she never will choose $y$ instead of $x$; lack of information enabling her to evaluate all the aspects of the alternatives could be a clear motive).

In this work the certain part of the basic preferences is taken as a primitive in the decision process. It is understood to be the immutable part of the individual's present preferences, and consequently, the known portion of her future preferences. This part of her tastes is represented by means of a binary relation $P$, which is only required to be asymmetric, but not necessarily negatively transitive, or complete, in accordance with its nature of total certainty. Sometimes it is quite clear that $x$ is better than $y$, and then we write $x P y$; in other cases it is not possible to assert this so clearly: even if there is a preference for $x$, this is not absolutely definite, therefore, $x$ and $y$ are not related through $P$. 
The structure of $P$ allows for the decision maker being unable to compare certain pairs of elements with absolute certainty, and for her preference between them being only approximate, allowing for the possibility of reversal. Also, it could happen that the decision maker might be so sure in her tastes that she could order all the alternatives by means of $P$, that is, it is possible for $P$ to be not only asymmetric, but also a negatively transitive relation. Likewise, the possibility is also admitted of the degree of uncertainty being great enough to consider $P$ empty. Of course the structure of $P$ includes cases in which it is, for example, a partial order, or a semiorder.

The above distinction fits in very well with the two notions introduced by Harsanyi [7]: The preferences such as they are at present or "actual preferences", and the "well-informed preferences", those hypothetical preferences the decision maker would have if she had all the relevant information about the alternatives and if she made use of it. In the model, $P$ represents the part of the well-informed preferences that the decision maker knows at present (at the moment of the evaluation of the opportunity sets).

The information attached to $P$ makes it possible to establish channels for the consistency which, in terms of preference for flexibility, must be maintained by the decision maker's comparisons of opportunity sets. In particular, preferences on opportunity sets are supposed to satisfy a minimal property: the addition of an alternative to a given set only implies a strict improvement if there is no other opportunity in the set which, by means of $P$, surpasses the one added (it is undoubtedly better); otherwise, the additional alternative does not contribute any flexibility to the choice, and in this sense, the enlarged set is considered to be indifferent to the original one.

The described condition establishes restrictions only on comparisons between sets which are enlargements (reductions) one of the other. Not in vain, have we tried to keep the condition free of any meaning beyond the mere concept of flexibility in choice. In its strictest sense, only sets related by means of inclusion would be susceptible to having this characteristic of providing "more (less) flexibility in the election than" the one contained (containing). This condition will not, therefore, be generally sufficient to obtain complete orderings on the opportunity sets. The actual extent of the property lies in the fact that it 
restricts the cases in which set enlargements actually increase their flexibility. In fact, there is a wide range of incomplete orders which verify the axiom, but this paper will focus mainly on those which are complete. Also, the proposed formalization makes it possible to experiment with the connection between the degree of uncertainty in the agent's tastes, and her wish for flexibility when choosing sets.

Consequently, the paper is set out as follows: Section 2 contains the notation and proposes a definition of the relations on sets which are consistent in terms of preference for flexibility with a given preference $P$. Section 3 holds the main results of the paper; among them, an axiomatic characterization of the class of relations over sets defined previously; and a representation theorem for the complete orderings which are consistent with a certain $P$. In Section 4 we present some special examples of orderings, taken from the freedom of choice literature, which can be interpreted as particular cases of consistency in terms of preference for flexibility. Section 6 contains some final remarks.

\section{Notation and definitions.}

$\Re\left(\Re_{+}\right)$will denote the set of all the real numbers (all the positives).

Let $X$ be a finite set of alternatives, and $\# X=n ; 2^{X}$ will represent the set of all the non-empty subsets of $X$. Sometimes, we will refer to the elements of $X$ as the "basic alternatives".

Let $P \subseteq X^{2}$ be a binary relation defined on $X$, it will be denoted $x P y$ when $(x, y) \in P$. In the present context, $P$ represents the absolutely certain part of the agent's preferences, and must be read as "is undoubtedly better than". According to this, $P$ will be said to be a certain preference relation, and will be supposed to be asymmetric.

Let $\subset 2^{X} \times 2^{X}$ be a binary relation defined on $2^{X}$. As usual, $A \quad B$ will indicate that "the set $A$ is preferred to $B$ ". At first, no particular structure will be imposed on , although, the paper will concentrate mainly on the case in which is an asymmetric and negatively transitive binary relation, and 
then, defined by: $A \quad B$ if $\neg\left(\begin{array}{ll}B \quad A\end{array}\right)$, where $\neg$ denotes logical negation, is a complete and transitive ordering on $2^{X}$, and the associated relation $\sim$ is an equivalence relation.

The formal goal of this work is to investigate possible binary relations on subsets of $X$ which are consistent in terms of preference for flexibility with the relation $P$ : We express this by the following definition:

Definition : Let $P$ be an asymmetric binary relation defined on $X$. It will be said that a binary relation $\subset 2^{X} \times 2^{X}$ is $P$-consistent in terms of preference for flexibility if it satisfies the following condition:

$$
\forall A \in 2^{X}, \forall x \in X / A,\left\{\begin{array}{l}
A \cup\{x\} \sim A \leftrightarrow \exists a_{i} \in A \text { s.t. } a_{i} P x \\
A \cup\{x\} \succ A
\end{array}\right.
$$

\section{Main results.}

First, we present and propose an axiomatic characterization of the relations over sets which are consistent in terms of flexibility with a preference $P$ : For this we consider the following axioms:

\section{Restricted Monotonicity (RM)}

$$
\forall x, y \in X, x P y \text { implies }\{x, y\} \sim\{x\} \text {, and } \neg(x P y) \text { implies }\{x, y\} \quad\{x\} .
$$

\section{Indifference Consistency: (IC)}

$\forall x \in X, \forall A, B \in 2^{X}$, s.t. $x \in X / A \cup B$ and $A \subseteq B, \quad A \cup\{x\} \sim A \rightarrow B \cup\{x\} \sim B$.

\section{Strict Preference Consistency: (SPC)}

$$
\forall x \in X, \forall A, B \in 2^{X} \text { s.t. } x \in X / A \cup B, \quad[A \cup\{x\} \quad A \text { and } B \cup\{x\} \quad B] \rightarrow A \cup B \cup\{x\} \quad A \cup B .
$$


(RM) is also used in Arlegi and Nieto [1]. It is related to the axiom of Strong Monotonicity (SM), used by Pattanaik and Xu [13] and Bossert, Pattanaik and Xu [5], according to which, for any $x, y \in X$, $\{x, y\} \quad\{x\}$; and which is used in a freedom of choice context. (RM) reinterprets (SM) in a context of preference for flexibility. By (RM), we restrict the cases in which value is attached to having a wider choice, and we consider that this happens only when there is some doubt in the basic preferences.

(IC) establishes that, if an alternative does not add any value to a given set $A$, then neither does it to a set which contains $A$. This property is related to the standard theory of consumer behavior. (SPC) states that, if an additional alternative increases the flexibility of a pair of sets, then it should likewise affect the union of those sets.

Theorem 1: Let be a binary relation defined on $2^{X}$, and let $P$ be an asymmetric binary relation defined on $X . \quad$ is $P$-consistent in terms of preference for flexibility (it satisfies (2.1)) if and only if satisfies (RM), (IC) and (SPC).

Proof: It is easy to check that, if satisfies (2.1), then it also satisfies (RM), (IC) and (SPC). We will only prove the inverse implication: Let $A \subseteq X, \# A=m$, and let $x \in X / A$ : If there exists $a_{i} \in A$ such that $a_{i} P x$, then, by (RM), $\left\{a_{i}, x\right\} \sim\left\{a_{i}\right\}$. As $\left\{a_{i}\right\} \subseteq A$, by (IC), $A \cup\{x\} \sim A$

If there does not exist $a_{i} \in A$ such that $a_{i} P x$, then, by (RM), $\forall a_{i} \in A\left\{a_{i}, x\right\} \quad\left\{a_{i}\right\}$, which by (SPC) implies $\left\{a_{1}\right\} \cup\left\{a_{2}\right\} \cup\{x\} \quad\left\{a_{1}\right\} \cup\left\{a_{2}\right\}$. Also by (SPC), the last relation implies in its turn $\left\{a_{1}\right\} \cup\left\{a_{2}\right\} \cup\left\{a_{3}\right\} \cup\{x\} \quad\left\{a_{1}\right\} \cup\left\{a_{2}\right\} \cup\left\{a_{3}\right\}$. By repeating as often as necessary, we have $\left\{a_{1}\right\} \cup\left\{a_{2}\right\} \cup \ldots \cup\left\{a_{n}\right\} \cup\{x\} \quad\left\{a_{1}\right\} \cup\left\{a_{2}\right\} \cup \ldots \cup\left\{a_{m}\right\}$, that is, $A \cup\{x\} \quad A$

(2.1) only establishes conditions of consistency with $P$ in cases of enlargements (reductions) in sets. This does not greatly restrict the structure of the possible orders on the opportunity sets; in fact, intransitive relations or clearly incomplete relations on opportunity sets can satisfy (2.1). From now on, 
the paper focuses mainly on those orders which satisfy (2.1) and are at the same time complete and transitive (complete preorders or "complete orderings").

This does not mean that we assume that the natural way to compare sets is with a complete and transitive preference. The previous result shows, in a way, that incompleteness in the basic preferences leads to incompleteness in the preferences over sets. The question now is: given a complete and transitive way to order opportunity sets (as in the freedom of choice models), is it possible to interpret it in terms of flexibility, that is, as a $P$-consistent ordering?

Definition. Let $P$ be an asymmetric binary relation defined on $X$. A binary relation on opportunity sets will be said to be a P-consistent ordering of opportunity sets in terms of preference for flexibility if it is complete and transitive, and satisfies (2.1) with respect to $P$.

Remark: It is easy to prove that, if $P$ is an asymmetric binary relation defined on $X$, and is a $P$ consistent ordering of opportunity sets, then $P$ is transitive.

As justified before, unlike Kreps, we try to formalize the idea of preference for flexibility in terms of the basic preferences, represented by the binary relation $P$. However, there is some relation between Kreps' approach and the one adopted in this paper.

Kreps [10] proposes a representation theorem for the preference for flexibility, but with no formal remission to preferences on the alternatives individually considered. The ex-ante conditions that he imposes on orderings of sets to assert that they display preference for flexibility are the following:

(3.1) $\forall A, B \in 2^{X}, A \subseteq B \rightarrow B \quad A$

(3.2) $\forall A, B, C \in 2^{X}, A \sim A \cup B \rightarrow \forall C \in 2^{X}, A \cup C \sim A \cup B \cup C$

That is, enlarging an opportunity set never makes things worse for the decision maker ((3.1)), and if a set $B$ does not add any additional value to another set $A$, then nor will it do so to a different set 
containing A ((3.2)). Therefore, Kreps concentrates on representing orderings which satisfy (3.1) and (3.2). Kreps' representation theorem establishes in advance one variable $s$ (the state of the individual's possible tastes or preferences), and one cardinal utility function dependent on $s, \quad U: X \times S \rightarrow \Re$, so that orderings on $2^{X}$ satisfy (3.1) y (3.2) if and only if there exists a finite set $S$, and a function $U: X \times S \rightarrow \Re$, such that the function $v: 2^{X} \rightarrow \Re$ defined by $v(A)=\sum_{\mathrm{s} \in \mathrm{S}}\left[\max _{x \in A} U(x, s)\right] \forall A \in 2^{X}$, represents . According to this representation, whenever the decision maker orders the opportunity sets satisfying properties (3.1) and (3.2), we may interpret that there is an underlying set of utility functions representing different orderings on $X$, orderings which are contingent to different possible states of the world (possible states of future preferences). Then, when the agent evaluates an opportunity set, she is behaving as if she were adding up the different maximal utilities she could reach with the elements of the set for each state $s_{i}$ of $S$.

The following Lemma relates the class of complete $P$-consistent orderings according to a given $P$, with the class of orderings represented by Kreps:

Lemma 1: Let be a complete and transitive binary relation defined on $2^{X}$, and let $P$ be an asymmetric binary relation defined on $X$. If is $P$-consistent in terms of preference for flexibility, then it satisfies (3.1) and (3.2).

Proof: (3.1) is straightforward from condition (2.1). To prove that (3.2) is also satisfied, note that, if is $P$-consistent in terms of preference for flexibility, then $A \sim A \cup B$ implies that $\forall b_{i} \in B \backslash A$ there exists $a_{i} \in A$ such that $a_{i} P b_{i}$; otherwise, by (2.1), $A \cup\left\{b_{i}\right\} A$, and by the monotonic character of and transitivity, $A \cup B \quad A$, which is absurd. Therefore, if $\forall b_{i} \in B \backslash A$ there exists $a_{i} \in A$ such that $a_{i} P b_{i}$, then $\forall b_{i} \in B \backslash A$ there exists $a_{i} \in A \cup C$ such that $a_{i} P b_{i}$; Take $b_{1}, \ldots, b_{n} \in B \backslash A$. By (2.1) $A \cup C \cup\left\{b_{1}\right\} \sim A \cup C$. For the same reason $A \cup C \cup\left\{b_{1}\right\} \cup\left\{b_{2}\right\} \sim A \cup C \cup\left\{b_{1}\right\}$, and by transitivity $A \cup C \cup\left\{b_{1}\right\} \cup\left\{b_{2}\right\} \sim A \cup C$. By repeating as often as necessary we reach $A \cup C \cup B \sim A \cup C$. 
In other words, although the starting point in our approach is quite different to the one in Kreps' work, the class of orderings consistent in terms of preference for flexibility with a given preference $P$ is representable à la Kreps. However, the inverse implication is not true in general. In particular, if a complete ordering satisfies (3.1) and (3.2), this does not imply that it satisfies (RM) or (SPC).

\section{Examples:}

- Let $X=\{x, y, z\} ; P=[(x, y)]$, and let $\quad$ be defined by: $\forall A, B \in 2^{X}, A \quad B \leftrightarrow \# A \geq \# B$. It is easy to check that this ordering ("counting" the alternatives) satisfies (3.1) and (3.2), (and also (IC) and (SPC)), but not (RM).

- Let $X=\{x, y, z\}$, and let defined by: $=[\{x, y, z\} \sim\{x, y\}] \quad[\{x, z\} \sim\{y, z\}] \quad[\{x\} \sim\{y\} \sim\{z\}]$. satisfies (3.1) and (3.2), but not (SPC), because $\{x, z\} \quad\{x\} ;\{y, z\} \quad\{y\}$, but $\{x, y, z\} \sim\{x, y\}$.

In the first example, the ordering is not consistent with the given relation $P$, but could be consistent with another different asymmetric relation $P$ (in fact it is consistent with an hypothetical $P^{\prime}=\varnothing$ ). The second example is in a way more relevant, as it is impossible to find any asymmetric relation $P$ on $X$ such that is consistent with it: According to the definition of $P$-consistency, $\{x, y, z\} \sim\{x, y\}$ would imply $x P z$ or $x P y$, and this would imply $\{x, z\} \sim\{x\}$ or $\{y, z\} \sim\{y\}$.

This point might lead us to investigate the necessary and sufficient axiomatic conditions for a complete ordering of opportunity sets for there to exist an asymmetric relation $P$ which parametrizes it in terms of flexibility. The following theorem answers this question. For this, the two following axioms are considered:

Weak Simple Monotonicity (WSM): $\forall x, y \in X,\{x, y\} \quad\{x\}$

Simple Relevance (SR): $\forall x, y \in X,\{x, y\} \quad\{x\}$ or $\{x, y\} \quad\{y\}$ 
(WSM) is a weak version of (RM). It claims that never is the availability of two opportunities worse than that of one of them alone. (SR) imposes, for any pair of elements, the existence of at least one which is relevant, that is, which contributes to the flexibility.

Theorem 2. Let be a binary relation defined on $2^{X}$. There exists an asymmetric binary relation $P$ on $X$ such that is $P$-consistent in terms of preference for flexibility if and only if satisfies (WSM), (SR), (IC) and (SPC).

Proof: It is straightforward that, given an asymmetric relation $P,(\mathrm{RM})$ implies (WSM) and (SR). Therefore, the proof of the sufficient part of Theorem 2 is provided by the proof of the sufficient part of Theorem 1. For the necessary part, we must prove that if a binary relation satisfies the axioms, then we can find an asymmetric relation $P$ such that $\quad$ is $P$-consistent. By (WSM), $\forall x, y \in X,\{x, y\} \quad\{x\}$ or $\{x, y\} \sim\{x\}$. We will show that, in particular, is consistent with the relation on $X$ defined by: $P=\{(x, y)$ such that $\{x, y\} \sim\{x\}\}$ : (From (SR) we have that $P$ defined as above is an asymmetric relation). Moreover, $\quad$ is $P$-consistent in terms of preference for flexibility. For that we must prove:

(i): $\forall A \in 2^{X}, \forall x \in X / A, \exists a_{i} \in A$ such that $a_{i} P x \rightarrow A \cup\{x\} \sim A$

and (ii): $\forall A \in 2^{X}, x \in X / A, \nexists a_{i} \in A$ such that $a_{i} P x \rightarrow A \cup\{x\} \quad A$

(i): Let $A \in 2^{X}, x \in X / A$. If there exists $a_{i} \in A$ such that $a_{i} P x$, then by definition $\left\{a_{i}, x\right\} \sim\left\{a_{i}\right\}$. By (IC), this implies $A \cup\{x\} \sim A$. (ii): Let $A \in 2^{X}, x \in X / A$. If there does not exist any $a_{i} \in A$ such that $a_{i} P x$, then, $\forall a_{i} \in X, \neg\left(\left\{a_{i}, x\right\} \sim\left\{a_{i}\right\}\right)$, which by (WSM) implies that $\forall a_{i} \in X,\left\{a_{i}, x\right\} \quad\left\{a_{i}\right\}$. Let $i=1, \ldots, \# A$. If \#A=1 we are done. Otherwise, $\left\{a_{1}, x\right\} \quad\left\{a_{1}\right\}$ and $\left\{a_{2}, x\right\} \quad\left\{a_{2}\right\}$, which by (SPC) implies $\left\{a_{1}, a_{2}, x\right\} \quad\left\{a_{1} a_{2}\right\}$. If $\# A>2,\left\{a_{3}, x\right\} \quad\left\{a_{3}\right\}$, which again by (SPC) implies $\left\{a_{1}, a_{2}, a_{3}, x\right\} \quad\left\{a_{1}, a_{2}, a_{3}\right\}$. By repetition, we reach $A \cup\{x\} \quad A$. 
Notice that Theorem 2 shows the condition for any binary relation on $2^{X}$ to exist an asymmetric relation $P$ which parametrizes it, and not only for complete orderings of sets. Theorem 2 has some formal analogies with the well known results of Sen [16] concerning rational choice. He imposes conditions, as $\alpha$ or $\gamma$, on the choice functions to assert that certain binary relations between the individual alternatives rationalize them. Theorem 2 imposes certain conditions on the set orderings sufficient to assert that there exists a binary relation that rationalizes those orderings, but in terms of flexibility. In that sense, the induced relation $P=\{(x, y)$ such that $\{x, y\} \sim\{x\}\}$, used in the proof, plays the role played by the revealed preference in the standard rationality models.

Theorem 2 shows no evident formal relationship with Kreps' characterization. But we know by Lemma 1 that such a relationship exists: given an asymmetric relation $P$, the set of $P$-consistent orderings are a subset of the orderings characterized by Kreps (but not the reverse). Then, it is worth posing the following question: Is it possible to characterize the particular form of Kreps' representation function, for the particular case of the $P$-consistent orderings? This point leads us to the following theorem of representation in terms of $P$ :

Theorem 3. Let $X$ be a finite set of alternatives, let $P$ be an asymmetric binary relation defined on $X$, and let be a complete and transitive ordering defined on $2^{X}$, then:

is $P$-consistent in terms of preference for flexibility if and only if there exists a finite set $S$, and a function $U: X \times S \rightarrow \Re$, such that $v(A)=\sum_{\mathrm{s} \in \mathrm{S}}\left[\max _{x \in A} U(x, s)\right] \forall A \in 2^{X}$ represents , and such that $U$ satisfies the following property with respect to $P$ :

$$
\forall A \in 2^{X}, \forall a_{i} \in A, a_{i} \in\left\{\max _{P}(A)\right\} \leftrightarrow \exists s \text { s.t. } U\left(a_{i}, s\right)>U\left(a_{j}, s\right) \forall a_{j} \in A, a_{j} \neq a_{i}
$$

Proof: By Lemma 1, if is P-consistent, it satisfies (3.1) and (3.2); hence, according to the representation theorem of Kreps [10], there exists a finite set $S$ and a function $U: X \times S \rightarrow \Re$, such that 
$\forall A \in 2^{X}, v(A)=\sum_{\mathrm{s} \in \mathrm{S}}\left[\max _{x \in A} U(x, s)\right]$ represents . We must prove that verifies (3.3) with respect to $P$. Let us suppose that this is not true; then, there are two possibilities. The first one is that for some $A \in 2^{X}$, there exists $a_{i} \in\left\{\max _{p}(A)\right\} \quad$ such that $\forall s_{j} \in S, \quad \exists a_{j} \neq a_{i}, \quad a_{j} \in A, \quad$ and $U\left(a_{j}, s\right) \geq U\left(a_{j}, s\right) ; \quad$ in that case, $\sum_{\mathrm{s} \in \mathrm{S}}\left[\max _{a_{j} \in A /\{a i\}} U\left(a_{j}, s\right)\right]=\sum_{\mathrm{s} \in \mathrm{S}}\left[\max _{a_{i} \in A} U\left(a_{i}, s\right)\right]$, and if $v$ represents $\quad$, then $A /\left\{a_{i}\right\} \sim A$, and consequently is not $P$ consistent. The second possibility is that for some $A \in 2^{X}$, there exists $a_{i} \notin\left\{\max _{P}(A)\right\}$, and $s \in S$, such that $\forall a_{j} \in A, a_{j} \neq a_{i}, U\left(a_{i}, s\right)>U\left(a_{j}, s\right)$; but if $a_{i} \notin\left\{\max _{P}(A)\right\}$, in this case there exists $a_{j} \in A$ such that $a_{j} P a_{i}$. Let us take the set $\left\{a_{i}, a_{j}\right\}$. By hypothesis and by definition of $v, v\left(\left\{a_{i}, a_{j}\right\}\right)>v\left(\left\{a_{j}\right\}\right)$. If $v$ represents, then $\left\{a_{i}, a_{j}\right\} \quad\left\{a_{j}\right\}$, therefore $\quad$ is not $P$-consistent.

On the other hand, we must prove that if there exists a finite set $S$, and a function $U: X \times S \rightarrow \Re$ satisfying (3.3), and such that the function $v$ represents , then is $P$-consistent. Again, in order to demonstrate this part of the proof, it is sufficient to prove the following:

(i) $\forall A \in 2^{X}, \forall x \in X / A, \exists a_{i} \in A$ such that $a_{i} P x \rightarrow A \cup\{x\} \sim A$

and (ii) $\forall A \in 2^{X}, \forall x \in X / A, \nexists a_{i} \in A$ such that $a_{i} P x \rightarrow A \cup\{x\} \quad A$

(i): Let $A \in 2^{X}, x \in X / A$. If there exists $a_{i} \in A$ such that $a_{i} P x$, then $x \notin\left\{\max _{P}(A \cup\{x\}\}\right)$. By (3.3) it is implied that, $\forall s \in S$, there exists $a_{j} \in A$, such that $U\left(a_{j}, s\right) \geq U(x, s)$, consequently $\sum_{\mathrm{s} \in \mathrm{S}}\left[\max _{a \in A} U\left(a_{i}, s\right)\right]=\sum_{\mathrm{s} \in \mathrm{S}}\left[\max _{a i \in A \cup\{x\}} U\left(a_{i}, s\right)\right]$, therefore $A \cup\{x\} \sim A$.

(ii): Let $A \in 2^{X}, x \in X / A$. If there is no $a_{i} \in A$ such that $a_{i} P x$, then $x \in\left\{\max _{P}(A \cup\{x\}\}\right.$. This implies that there exists $s \in S$ such that $U(x, s)>U\left(a_{i}, s\right) \forall a_{i} \in A$, consequently $\sum_{\mathrm{s} \in \mathrm{S}}\left[\max _{a i \in A} U\left(a_{i}, s\right)\right]<\sum_{\mathrm{s} \in \mathrm{S}}\left[\max _{a i \in A \cup\{x\}} U\left(a_{i}, s\right)\right]$, therefore $A \cup\{x\} \quad A$.

Interpretation of Theorem 3: In our approach, the relation $P$ over the alternatives, and the consistency condition (2.1) with respect to $P$, are the primitives in the model. From them, we arrive at orderings on 
opportunity sets which, by Lemma 1, are within the general framework of Kreps, but which are parametrized by the preference $P$. This reveals that any $P$-consistent ordering must be also representable in terms of utility contingent functions, and indicates that there must be some specific connection between the structure of $P$ and the structure of the utility functions; that is, the requirement of consistency with $P$ must unavoidably constrain the type of representation. This restriction is given by property (3.3), which demands that one alternative is not dominated by means of $P$ by any other in the its set, if and only if there exists a state $s \in S$ where the alternative is the best in the set.

Property (3.3) characterizes the particular form of Kreps' representation functions in the case of $P$ consistent rankings: Given a relation $P$, given a $P$-consistent ordering, and given a Kreps-type function representing it, then, the function must verify (3.3). On the other hand, given a relation $P$, and given a Kreps-type function satisfying (3.3) according to $P$, then such a function represents a $P$-consistent ordering of the opportunity sets. This means that $P$ parametrizes, not only the class of $P$-consistent orderings, but also, the set of Kreps-type functions which can characterize them.

Furthermore, (3.3) implies that, given $P$, and one function like v representing a $P$-consistent ordering of opportunity sets, then we can define it as: $x P y \leftrightarrow U(x, s) \geq U(y, s) \forall s$, with strict inequality for some $s$. However, it is possible to prove that the inverse implication is not true: given $P$, it is not true that any function $U$ satisfying $x P y \leftrightarrow U(x, s) \geq U(y, s) \forall s$, with strict inequality for some $s$, is useful to represent an ordering consistent in terms of preference for flexibility with $P$. We can then propose the following

Corollary: Let $X$ be a finite set of alternatives, let $P$ be an asymmetric relation defined on $X$, let be a $P$-consistent order defined on $2^{X}$, and let be a finite set $S$, and a function $U: X \times S \rightarrow \Re$, satisfying (3.3) and such that $v(A)=\sum_{\mathrm{s} \in \mathrm{S}}\left[\max _{x \in A} U(x, s)\right] \forall A \in 2^{X}$ represents . Let us define a function $\phi: X \rightarrow \Re$, such that: $\forall x, y \in X, \phi(x)>\phi(y) \leftrightarrow U(x, s) \geq U(y, s) \forall s$, with strict inequality for some $s$.

Then, $\phi$ (weakly) represents $P$; that is, $\forall x, y \in X \quad x P y \rightarrow \phi(x)>\phi(y)$. 


\section{Examples.}

Below we study different criteria for ordering opportunity sets taken from the related literature, and in particular, from the freedom of choice literature. We analyze how to interpret them in terms of preference for flexibility, that is, they are described as consistent rankings according to a given relation $P$, and, therefore, we propose ways to represent them in the form established by Theorem 3 .

Proposition 1. Let $P$ be an asymmetric binary relation defined on $X$ such that $\forall x, y \in X ; x \neq y ; x P y$ or $y P x$. Let be a complete ordering defined on $2^{X}$. Then is $P$-consistent in terms of preference for flexibility if and only if is the standard indirect utility criterion $\left(A \quad B \leftrightarrow \max _{\mathrm{R}}(A) \operatorname{Rmax}_{\mathrm{R}}(B)\right.$ ) (where $R$ is the weak preference relation associated to $P$, which is complete and transitive)

Proof. The necessary part of the implication is quite straightforward; we will prove only the sufficient part: We must prove that, given $P$ as defined, if $\quad$ is $P$-consistent, then $\quad$ is the standard indirect utility criterion. For this we have to prove:

(i): $\max _{\mathrm{R}}(A) P \max _{\mathrm{R}}(B) \rightarrow A \quad B$

(ii): $\max _{\mathrm{R}}(A) \operatorname{Imax}_{\mathrm{R}}(B) \rightarrow A \sim B($ where $I$ denotes the indifference relation associated to $P$ )

(i): Given the formal structure of $P, \forall A \in 2^{X}, \max _{\mathrm{R}}(A)$ exists and is unique. Idem with $\max _{\mathrm{R}}(B)$. Let $a_{1}=\max _{\mathrm{R}}(A), b_{1}=\max _{\mathrm{R}}(B)$. By hypothesis, $a_{1} P b_{1}$. As $\quad$ is a $P$-consistent binary relation, and $P$ is asymmetric, then $\left\{a_{1}, b_{1}\right\} \sim\left\{a_{1}\right\} \quad\left\{b_{1}\right\}$. As $P$ is connected, $\forall a_{\mathrm{i}} \in A, a_{1} \neq a_{\mathrm{i}}, a_{1} P a_{i}$; and $\forall b_{\mathrm{i}} \in A, b_{1} \neq b_{i}$, $b_{1} P b_{i}$. Hence, by condition (2.1) repeatedly applied, $\left\{a_{1}\right\} \sim A$, and $\left\{b_{1}\right\} \sim B$. As is a complete ordering, $A B$. 
(ii): Let $a_{1}=\max _{\mathrm{R}}(A), b_{1}=\max _{\mathrm{R}}(B)$. As $P$ is connected, $\max _{\mathrm{R}}(A) \operatorname{Imax}_{\mathrm{R}}(B)$ implies $a_{1}=b_{1}$. By reflexivity of $\sim,\left\{a_{1}\right\} \sim\left\{b_{1}\right\}$, then, as in (i), by property (2.1), $\left\{a_{1}\right\} \sim A$ and $\left\{b_{1}\right\} \sim B$. And by transitivity and completeness of $\quad, A \sim B$.

Proposition 2. Let $P$ be an asymmetric, transitive and connected binary relation defined on $\mathrm{X}$, and let be a complete ordering defined on $2^{X}$. Then, is $P$-consistent in terms of preference for flexibility if and only if there exists a set $S$, and a function $U: X \times S \rightarrow \Re$, such that $S=\left\{s_{1}\right\} ; U:\left(X, s_{1}\right) \rightarrow \Re$ represents $P$; and $v(A)=\sum_{\mathrm{s} \in \mathrm{S}}\left[\max _{x \in A} U(x, s)\right] \forall A \in 2^{X}$ represents .

Proof: By Proposition 1, is the indirect utility criterion, that is, $\forall A, B \in 2^{\mathrm{X}}, A \quad B \leftrightarrow \max _{\mathrm{R}}(A) \operatorname{Rmax}_{\mathrm{R}}(B)$. Then, let $U: X \times S \rightarrow \Re$ such that $S=\left\{s_{1}\right\}$ and $U:\left(X, s_{1}\right) \rightarrow \Re$ represents $P \quad(\forall x, y \in X, \quad x P y \leftrightarrow$ $\left.\leftrightarrow U\left(x, s_{1}\right)>U\left(y, s_{1}\right)\right)$ : By the definition of function $v, \forall A \in 2^{X}, v(A)=U\left(\max _{\mathrm{R}}(A), s_{1}\right)$, as $\quad$ is the indirect utility criterion, $\forall A, B \in 2^{X}, A B \leftrightarrow \max _{\mathrm{R}}(A) \operatorname{Rmax}_{\mathrm{R}}(B)$; as $U$ represents $P$, $\max _{\mathrm{R}}(A) R \max (B) \leftrightarrow$ $\leftrightarrow U\left(\max _{\mathrm{R}}(A), s_{1}\right) \geq U\left(\max _{\mathrm{R}}(B), s_{1}\right) ;$ and by the definition of $v, \quad U\left(\max _{\mathrm{R}}(A), s_{1}\right) \geq U\left(\max _{\mathrm{R}}(B), s_{1}\right) \leftrightarrow$ $\leftrightarrow v(A) \geq v(B)$, that is, $v$ represents .

On the other hand, let the relations $P$, and the functions $U, v$ defined in the hypothesis, such that $v(A)=\sum_{\mathrm{s} \in \mathrm{S}}\left[\max _{x \in A} U(x, s)\right] \forall A \in 2^{X}$ represents ; we must prove that is $P$-consistent. For that we must prove:

(i) $\forall A \in 2^{X}, \forall x \in X / A, \exists a_{i} \in A$ such that $a_{i} P x \rightarrow A \cup\{x\} \sim A$. and (ii) $\forall A \in 2^{X}, \forall x \in X / A, \nexists a_{i} \in A$ such that $a_{i} P x \rightarrow A \cup\{x\} \quad A$.

(i): By the definition of functions $U$ and $v$, if there exists $a_{i} \in A$ such that $a_{i} P x$, then $v(A \cup\{x\})=v(A)$. If $v$ represents , then $A \cup\{x\} \sim A$. (ii): By the formal structure of $P$, if there does not exist $a_{i} \in A$ such 
that $a_{i} P x$, then $x P a_{i} \forall a_{i} \in A$. That is, $\max _{\mathrm{R}}(A \cup\{x\})=x$ and $x P \max _{\mathrm{R}}(A)$. Therefore, by the definition of $U$ and $v, v(A \cup\{x\})>v(A)$. If $v$ represents , then $A \cup\{x\} \quad A$.

From Propositions 1 and 2 we have that, when there is no uncertainty (the binary relation $P$ relates all the possible pairs), the rule obtained is the one based on the indirect utility of the opportunity sets. Proposition 2 illustrates this fact, since it shows that such a situation can be interpreted as the existence of a unique state of the future preferences.

Proposition 3. Let $P$ be the empty binary relation defined on $X$, and let be a complete ordering defined on $2^{X}$. is $P$-consistent in terms of preference for flexibility if and only if it satisfies that $\forall A, B \in 2^{X}, B \subset A \rightarrow A \quad B$. The proof is quite straightforward, so it is omitted.

Many rules in the freedom of choice literature satisfy the monotonic feature of Proposition 3. We have selected some paradigmatic ones:

The cardinalist ranking of opportunity sets ( \#) is characterized and defined in Pattanaik and Xu [13] by: $\forall A, B \in 2^{X}, A \quad \# B \leftrightarrow \# A \geq \# B$.

The leximax rule $\left({ }_{L}\right)$ is defined in Bossert, Pattanaik and $\mathrm{Xu}$ [5] as follows: Let $\mathbf{R}$ be a complete ordering defined on $X$. Let $\mathrm{u}_{\mathbf{R}}: X \rightarrow \Re_{+}$such that $\forall x, y \in X, \mathrm{u}_{\mathbf{R}}(x) \geq \mathrm{u}_{\mathbf{R}}(y) \leftrightarrow x R y$. Let $\mathrm{v}_{\mathbf{R}}: 2^{X} \rightarrow \Re^{n}+$, $\mathrm{v}_{\mathbf{R}}(A)=\left[\mathrm{u}_{\mathbf{R}}\left(a_{1}\right), \mathrm{u}_{\mathbf{R}}\left(a_{2}\right), \cdots, \mathrm{u}_{\mathbf{R}}\left(a_{s}\right), 0^{n-s}\right] \forall A \in 2^{X}$. And let $\geq_{\mathrm{L}}$ be the lexicographic order on $\Re^{n}$. Then

$$
\forall A, B \in 2^{X}, A \quad{ }_{L} B \leftrightarrow \mathrm{v}_{\mathbf{R}}(A) \geq_{\mathrm{L}} \mathrm{v}_{\mathbf{R}}(B) .
$$

The cardinality-first lexicographic relation $\left({ }_{C F L}\right)$, and the preference-first lexicographic relation ( ${ }_{P F L}$ ) are defined as follows, also in Bossert, Pattanaik and $\mathrm{Xu}$ [5]: Let $\mathbf{R}$ be a complete ordering on $X$, 
$\forall A, B \in 2^{X}, A \quad{ }_{C F L} B \leftrightarrow[\# A>\# B]$ or $\left[\# A=\# B\right.$ and $\left.\max _{\mathrm{R}}(A) \mathbf{R} \max _{\mathrm{R}}(B)\right]$

$\forall A, B \in 2^{X}, A{ }_{P F L} B \leftrightarrow \max _{\mathrm{R}}(A) P \max _{\mathrm{R}}(B)$ or $\left[\max _{\mathrm{R}}(A) \mathbf{I} \max _{\mathrm{R}}(B)\right.$ and $\left.\# A \geq \# B\right]$

Corollary. If $P=\varnothing$ then $\#, \quad{ }_{L}, \quad L_{L C P}$ and ${ }_{L P C}$ are $P$-consistent in terms of preference for flexibility. To prove this, we must simply take into account that the four criteria satisfy that, $\forall A, B \in 2^{X}$, $B \subset A \rightarrow A \quad B$.

Proposition 4. Let $P$ be the empty binary relation defined on $X$, and let be a complete ordering defined on $2^{X}$. is $P$-consistent in terms of preference for flexibility if and only if there exists a set $S$, and a function $U: X \times S \rightarrow \Re$, such that $U$ satisfies (3.3), and $\forall x \in X$ there exists $s \in S$ such that $U(x, s)>U(y, s) \forall y \in X, y \neq x$; and $v(A)=\sum_{\mathrm{s} \in \mathrm{S}}\left[\max _{x \in A} U(x, s)\right] \forall A \in 2^{X}$ represents $\quad$.

Proof: By Theorem 3, given an asymmetric binary relation $P$, and a complete ordering which is $P$ consistent, then there exists a set $S$, and a function $U: X \times S \rightarrow \Re$, such that $v(A)=\sum_{s \in S}\left[\max _{x \in A} U(x, s)\right] \forall A \in 2^{X}$ represents , and such that function $U$ satisfies (3.3). We must then prove that, when $P$ is the empty relation, for any $x \in X$ there exists $s \in S$ such that $U(x, s)>U(y, s) \forall y \in X, y \neq x$ : For any $x \in X$, let $X \backslash\{x\}$. As $P$ is empty, $x \in \max _{\mathrm{P}}(X)$, hence, by (3.3) there exists $s \in S$ such that $U(x, s)>U(y, s) \forall y \in X, y \neq x$.

On the other hand, we must prove that, given the empty relation $P$; the complete ordering ; and the functions $U, v$ defined in the hypothesis, such that $v(A)=\sum_{\mathrm{s} \in \mathrm{S}}\left[\max _{x \in A} U(x, s)\right] \forall A \in 2^{X}$ represents , then is $P$-consistent. By these definitions, $\forall A \in 2^{X}, \forall x \in X / A, v(A \cup\{x\})>v(A)$, and if $v$ represents, this implies that, $\forall A \in 2^{X}, \forall x \in X / A, A \cup\{x\} \quad A$. Therefore, $\forall A, B \in 2^{X}, B \subset A \rightarrow A \quad B$. By Proposition 3, this implies that is $P$-consistent with the empty relation $P$. 
The case shown by Propositions 3 and 4 is at the opposite extreme from that of Propositions 1 and 2 . On the one hand, the complete absence of certainty, (displayed by the fact that $P$ is empty), gives full value to flexibility in choice: any additional alternative implies a strict improvement. On the other hand, while in Propositions 1 and 2 the absence of uncertainty was associated with a unique state of the future preferences, when the uncertainty is total, there must be, at least, as many states in $S$ as there are elements in $X$. Below we propose some examples to represent ${ }_{\#}, \quad{ }_{L}, \quad L_{L C P}$ and ${ }_{L P C}$, that may clarify the meaning of the previous arguments. The proofs of the examples are omitted.

Example 1: Let $S=\left\{s_{1}, s_{2} \ldots, s_{n}\right\}, X=\left\{x_{1}, x_{2} \ldots, x_{n}\right\}(\# S=\# X=n)$, and let $c$ be any positive constant value,

$$
\begin{aligned}
& \text { let } U: X \times S \rightarrow \Re \text { be defined by: } \forall x_{i} \in X, U\left(x_{i}, s_{j}\right)=\left\{\begin{array}{l}
c \text { if } j=i \\
0 \text { if } j \neq i
\end{array}\right. \\
& \text { then } v(A)=\sum_{\mathrm{s} \in \mathrm{S}}\left[\max _{x \in A} U(x, s)\right] \forall A \in 2^{X} \text { represents \#. }
\end{aligned}
$$

Example 2: Let $S=\left\{s_{1}, s_{2} \ldots, s_{n}\right\}, X=\left\{x_{1}, x_{2} \ldots, x_{n}\right\},(\# S=\# X=n)$. Let $\mathbf{R}$ be a complete ordering defined on $X$, let $X / \mathbf{I}=\left\{C_{1}, C_{2} \ldots, C_{m}\right\}$ be the quotient set determined by $\mathbf{I}$ such that $C_{m} \mathbf{P} C_{m-1} \mathbf{P} \cdots \mathbf{P} C_{1}$, and let $n_{m}=$ $=\max \left(\# C_{i}\right)$

Let $U: X \times S \rightarrow \Re$ be defined by: $\forall x_{i} \in \mathrm{C}_{k} \subseteq X, U\left(x_{i}, s_{j}\right)=\left\{\begin{array}{cc}\left(n_{m}+1\right)^{\mathrm{k}-1} & \text { if } j=i \\ 0 & \text { if } j \neq i\end{array}\right.$ then $v(A)=\sum_{\mathrm{s} \in \mathrm{S}}\left[\max _{x \in A} U(x, s)\right] \forall A \in 2^{X}$ represents $\quad L$.

Examples 1 and 2 satisfy the conditions of Proposition 4, but the different type of information about the agent's tastes finds a different expression when represented by means of function $U$. In the first example, there is no preference (either certain or "probable") on basic alternatives; therefore, any of them has an equal chance of being the best one in the future. Under the leximax rule there is a preference 
relation, $\mathbf{R}$, which can be interpreted as "uncertain" or "probable", and which reveals more about the agent's tastes. So, when representing by function $U$, any alternative can be the best one in future, but according to a consistent hierarchy in relation to $\mathbf{R}$.

Example 3: Let $X=\left\{x_{1}, x_{2} \ldots, x_{n}\right\}, S=\left\{s_{0}, s_{1} \ldots, s_{n}\right\},(\# S=n+1)$. Let $\mathbf{R}$ be a complete ordering defined on $X$; let $X / \mathbf{I}=\left\{C_{1}, C_{2} \ldots, C_{m}\right\}$ the quotient set determined by $\mathbf{I}$ such that $C_{m} \mathbf{P} C_{m-1} \mathbf{P} \cdots \mathbf{P} C_{1}$.

Let $U: X \times S \rightarrow \Re$ defined by: $\forall x_{i} \in \mathrm{C}_{k} \subseteq X, \quad U\left(x_{i}, s_{j}\right)= \begin{cases}k & \text { if } j=0 \\ n & \text { if } j=i \\ 0 & \text { if } j \neq 0, i\end{cases}$

then $v(A)=\sum_{\mathrm{s} \in \mathrm{S}}\left[\max _{x \in A} U(x, s)\right] \forall A \in 2^{X}$ represents $\quad C F L$.

Example 4. Let $X=\left\{x_{1}, x_{2} \ldots, x_{n}\right\}, S=\left\{s_{0}, s_{1} \ldots, s_{n}\right\},(\# S=n+1)$. Let $\mathbf{R}$ be a complete ordering defined on $X$; let $X / \mathbf{I}=\left\{C_{1}, C_{2} \ldots, C_{m}\right\}$ the quotient set determined by $\mathbf{I}$ such that $C_{m} \mathbf{P} C_{m-1} \mathbf{P} \cdots \mathbf{P} C_{1}$.

Let $U: X \times S \rightarrow \Re$ defined by: $\forall x_{i} \in \mathrm{C}_{k} \subseteq X, U\left(x_{i}, s_{j}\right)=\left\{\begin{array}{c}k \text { if } j=0 \\ 1 / n \text { if } j=i \\ 0 \text { if } j \neq 0, i\end{array}\right.$

then $v(A)=\sum_{s \in S}\left[\max _{x \in A} U(x, s)\right] \forall A \in 2^{X}$ represents $\quad P F L$.

\section{Summary and conclusions}

We have analyzed orderings on opportunity sets in order to formalize the concept of preference for flexibility. We are inspired by the interpretation of this notion as suggested by Koopmans [9] and Kreps [10]. Although the notion of preference for flexibility and that of preference for freedom of choice seem to be similar, there are some causal differences: in the numerous recent works about freedom of choice, the value of having more to choose from is not necessarily incompatible with preferences over the alternatives which are certain and complete and, therefore, these works appeal to the intrinsic value of the mere availability of more possibilities to choose from. Certainly, in some contexts, freedom of choice 
can be endowed with some value per se, but the motivation of the present work lies in the hypothesis that, often, insufficiently certain tastes or preferences can be the real underlying cause of the wish for more to choose from. This conjecture would justify the need to provide a coherent formalization of the relation between the preference for more opportunities (termed "preference for flexibility" under this interpretation) and uncertainty in tastes.

A reasonable approach to this problem can be achieved by looking at the very structure of the preferences over the basic alternatives, as a primitive in the decision procedure. Therefore, we are required to distinguish (in a return to the distinction proposed by Harsanyi) between those preferences over basic alternatives which are sufficiently certain, and those which are doubtful, and thereby, establish the subsequent modeling of a basic preferences structure to match such a distinction. We link preferences on opportunity sets with the preference over the basic alternatives by means of a minimal consistency condition (Condition (2.1)), and the result turns out to fall within the general domain of Kreps' proposal. Condition (3.3) establishes the connection between Kreps' approach and that of this paper.

Furthermore, it is noted how some well-known rules for ordering opportunity sets, also given as extensions of basic preferences, belong to the class of orderings proposed in this work, and precisely these rules can be interpreted as special cases of preference for flexibility, which are related to different degrees of uncertainty. This circumstance has its formal reflection in the fact that each of these rules arises from a particular form of the basic preferences structure, and also from particular forms to be represented by the Kreps' formula. The formal particularizations obtained in each case fit the main hypothesis of this paper, that is, that the value of having more to choose from is directly related to the degree of uncertainty, displayed by the "absolutely certain" basic preference. Precisely those rules where the availability of more opportunities is more highly valued (the decision maker displays more preference for flexibility), require, on the one hand, the "certain" part of the basic preferences to be "smaller", and on the other hand, when representing them, the existence of possible states of the future preferences which allow for their reversals. 
This paper has focused its attention on complete orderings on opportunity sets, which seem to be more appropriate for the purpose of political evaluation. Nevertheless, it would also be interesting to approach the analysis of incomplete orders, which, as hinted at by Sen ([19],p.19), could arise when trying to describe the preference for flexibility or for freedom of choice. In fact, the initial results in Section 3 are not constrained to the assumption that the orderings on sets are complete, so that, this opens many possibilities for further investigation.

Finally, there are some additional aspects related to comparisons of opportunity sets, studied in merely a few recent works, but which are worthy of more thorough research. For example, the incidence of variety between the alternatives within opportunity sets (Bavetta and Del Seta [3], Nehring and Puppe [11]), or the consequences of the presence of evaluation costs (Neme, Nieto and Quintas [12]). 


\section{References}

1. R. Arlegi and J. Nieto, "Preference for Flexibility: An Axiomatic Approach", Documentos de Trabajo del Departamento de Economía, D.T. 9602 (1996), Universidad Pública de Navarra.

2. K. Arrow, "A Note on Freedom and Flexibility”, in Basu, K., P. Pattanaik and K.Suzumura (eds.): 'Choice, Welfare and Development: A Festschrift in Honour of Amartya Sen', Oxford University Press.

3. S. Bavetta and M. Del Seta, "Rough Set Approximations and Freedom of Choice", Discussion Paper Series of the Centre for the Philosophy of the Natural and Social Sciences, DP 26/96. London School of Economics (1996).

4. W. Bossert, "A Leximax Ordering for Opportunity Sets", Waterloo Economic Series, Working Paper 9202 (1992), University of Waterloo.

5. W. Bossert, P. K. Pattanaik and Y. Xu, "Ranking opportunity sets: An axiomatic approach", $J$. Econ. Theory 63 (1994), 326-345.

6. N. Gravel, "Ranking opportunity sets on the basis of their freedom of choice and their ability to satisfy preferences: A difficulty”. Soc.Choice Welfare 15(3) (1998), 371-382.

7. J. C. Harsanyi, "Utilities, preferences and substantive goods", Soc.Choice Welfare 14 (1) (1997), 129-145.

8. M. Klemisch-Ahlert, "Freedom of Choice. A comparison of different rankings of opportunity sets", Soc. Choice Welfare 10 (1993), 189-207.

9. T. C. Koopmans, "On the flexibility of future preferences", In: M. W. Shelley and G. L. Bryan (eds.), Human Judgments and Optimality, Wiley, New York, (1964).

10. D.M. Kreps, "A representation theorem for "Preference for Flexibility", Econometrica, (1979), 565-577.

11. K. Nehring and C. Puppe, "A theroy of diversity", mimeo. University of Bonn. 
12. A. Neme, J. Nieto and L. Quintas, "Ranking Opportunity Sets: Freedom of Choice and the Cost of Information", Documentos de Trabajo del Departamento de Economía, D.T. 9601 (1996), Universidad Pública de Navarra.

13. P. K. Pattanaik and Y. Xu, "On Ranking Opportunity Sets in Terms of Freedom of Choice”, Rech. Econ. Louvain 56 (1990), 383-390.

14. C. Puppe, "Freedom of Choice and Rational Decisions", Soc. Choice Welfare 12 (2) (1995), 13753.

15. C. Puppe, "An axiomatic approach to 'Preference for freedom of choice"”, J. Econ. Theory 68 (1) (1996), 174-99.

16. A. K. Sen "Choice Functions and Revealed Preference”. Review of Economic Studies 38 (1971), 307-317.

17. A. K. Sen, “Commodities and Capabilities” North-Holland, Amsterdam. 1985.

18. A. K. Sen, "Freedom of choice: Concept and content". Europ. Econ. Rev. 32 (1988), 269-294.

19. A. K. Sen, "Welfare, Preference and Freedom". J . Econometrics 50 (1991), 15-29.

20. P. Suppes, "Maximizing Freedom of Decision: An axiomatic analysis". In G.Feiwel, ed., Arrow and the foundations of Economic Policy, New York: New York University Press (1987). 